\title{
Defectos en madera de eucalipto asociados a daños por heladas en Entre Ríos, Argentina
}

\section{Defects of eucalypt wood associated with frost damage in Entre Ríos, Argentina}

\author{
María de los Ángeles Garcíal* y Ciro Andrés Mastrandrea'
}

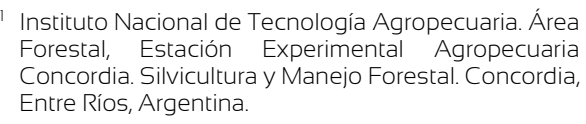

\section{RESUMEN}

En un rodal de Eucalyptus grandis afectado por heladas se estudió la asociación entre daños por heladas y defectos en la madera. Cuatro meses después de las heladas se clasificaron 40 árboles de 3 años en 3 clases de daño por heladas: sin daño aparente, ápice seco, toda la copa dañada; se les midió altura y diámetro normal (DAP) y dos años después se midieron nuevamente y se apearon 19 individuos de las 3 clases. En las dos primeras trozas se estimó el módulo de elasticidad dinámico (MOE) y en las tablas se registró presencia de médula, pudrición, grietas y kino. Los datos se analizaron con modelos mixtos, prueba LSD de Fisher, análisis de correspondencia múltiple y modelos lineales generalizados mixtos. En la primera medición los árboles sin daño aparente presentaban altura y DAP significativamente mayores a los demás. Dos años después de la primera medición los árboles que habían presentado toda la copa dañada mostraron valores de DAP significativamente menores. El MOE de las segundas trozas resultó significativamente mayor en árboles sin daño aparente comparados con los de toda la copa dañada. Solo se observó pudrición en árboles con daño visible, pero sin diferencias significativas en la proporción de tablas con pudrición en las dos clases. La asociación entre grietas y daño por heladas no fue significativa. El daño por heladas según la escala usada se asocia con pudrición en tablas y con el módulo de elasticidad de las trozas.

PALABRAS CLAVE: clasificación visual, frío, método no destructivo, módulo de elasticidad, plantaciones forestales, pudrición.

\section{ABSTRACT}

We studied the association of visible frost damage with wood defects of Eucalyptus grandis trees affected by frost. Four months after frosts occurrence we classified 40 trees from a three-years-old stand in three classes of damage: no visible frost damage; frozen top; completely frozen crown; at the same time we measured trees height $(\mathrm{H})$ and diameter at breast height $(\mathrm{DBH})$ and after two years we measured 19 trees of the three classes of frost damage again and then we cut them. We also assessed the dynamic modulus of elasticity (DMoE) of the basal and the second logs and recorded the presence of pith, rot, cracks and kino on timber boards. Trees with no apparent frost damage showed significantly higher height and DBH compared with trees from the other classes, whereas by the second measurement trees with completely frozen crown presented significantly lower DBH than those from the other classes. DMoE of second logs was significantly higher in trees with no apparent frost damage compared with trees with completely frozen crown. We found rot only in timber boards from trees with frost damage, but we found no significant differences in the number of timber boards with rot between the two classes with visible frost damage. Association of cracks with frost damage was not significant. The classes of damage used in this study allowed to detect rot on tables and to differentiate trees according to DMoE of logs.

KEYWORDS: visual classification, cold, non-destructive method, modulus of elasticity, plantation forests, rot. 


\section{INTRODUCCIÓN}

En la provincia de Entre Ríos (Argentina) las plantaciones de Eucalyptus grandis son frecuentemente afectadas por heladas invernales, que causan quemado de los márgenes de las hojas, daño de la yema apical, grietas en la corteza y, en algunos casos, la muerte de las plantas. Los daños se manifiestan en los brotes recientes y en la porción del fuste con diámetros menores y corteza más delgada (Prado, 1989; Gurskaya y Shiyatov, 2006; Schweingruber, 2007). En la madera se observan acebolladuras, grietas, rajaduras, bolsas de kino (el kino es una secreción de algunos eucaliptos, muy rica en polifenoles [Alonso-Peña, 2011]), anillos muertos, cambios en la densidad, pudrición (Pearce, 2001; Gurskaya y Shiyatov, 2006; Schweingruber, 2007). La pérdida del ápice o de toda la copa puede ocurrir varios meses después de la helada (Broschat, 2013), por lo cual los daños deben evaluarse de 2 meses a 6 meses luego de producido ese evento meteorológico (Crane, 2010).

En junio de 2012 se registraron las heladas más intensas de los últimos 80 años, desde el sur de la provincia de Corrientes hasta el norte de Entre Ríos, con temperaturas entre $-3,1{ }^{\circ} \mathrm{C}$ y $-5,7{ }^{\circ} \mathrm{C}$, comparables con las ocurridas en Concordia en junio de 1996 (-5,1 ${ }^{\circ} \mathrm{C}$, récord histórico de Concordia desde 1930) y julio de $2007\left(-4,8^{\circ} \mathrm{C}\right)$ en cuanto a intensidad y duración, fueron excepcionales por su extensión territorial, porque se produjeron antes de la fecha media de la primera helada meteorológica y porque se registraron en tres días consecutivos (Garrán y Garín, 2007). De la Peña, Flores y Ramos (2012) evaluaron las pérdidas económicas causadas por las heladas de 2012 sobre las plantaciones de eucalipto de la costa del río Uruguay y resaltan la dificultad para estimar las pérdidas económicas asociadas a defectos en la madera de los árboles afectados, ya que no existen cuantificaciones de daño por heladas en árboles en pie que permitan estimar el daño en la madera aserrada antes del apeo. Aserrar rollos provenientes de árboles afectados por heladas puede disminuir el rendimiento y la calidad de las tablas obtenidas por aserrado. Del Tufo (2012) indica que, al aserrar trozas provenientes de un lote afectado por heladas, 30\% de las tablas obtenidas fueron descartadas por presentar daño y menciona una pérdida de $9 \%$ a $12 \%$ del volumen total por deformaciones de los rollos. Defectos como rajaduras, venas y bolsas de kino y pudrición en tablas aserradas son admitidos en cierta cantidad y tamaño por diferentes reglas de tipificación de madera; la regla de tipificación de madera de Eucalptus grandis para la Mesopotamia (Shield y Mastrandrea, 2007) no admite rajaduras ni pudrición, debiéndose sanear o descartar las tablas con esos defectos. Lo anterior causa la disminución del factor de conversión y, por tanto, una pérdida económica. Sin embargo, no existe todavía un método para estimar los daños en la madera hasta que las trozas son aserradas.

\section{OBJETIVOS}

Evaluar la asociación entre clases visuales de daños por heladas en árboles en pie y defectos en la madera aserrada proveniente de árboles de cada clase de daños por heladas.

\section{MATERIALES Y MÉTODOS}

El estudio se situó en un rodal comercial de Eucalyptus grandis de 3 años de edad ubicado en el paraje Humaitá (Colón, Entre Ríos, Argentina), afectado en distinta intensidad por las heladas ocurridas en junio de 2012. En noviembre de 2012 se seleccionaron 40 árboles de ese rodal y se clasificaron, según una escala visual de daño por heladas, en tres clases: a) copa sana, sin daño aparente por heladas, b) ápice seco, resto de la copa sin daño por heladas, c) copa totalmente seca, rebrotando algunas ramas o el fuste (Fig. 1). A los árboles seleccionados y clasificados se les midió altura (H) y diámetro normal (DAP).

En 2014, dos años después de ocurridas las heladas, a los árboles clasificados en 2012 se les midió nuevamente altura y diámetro, y se seleccionaron y apearon 19 de ellos ( 5 de clase de daño a, 7 de clase de daño b y 7 de clase de daño c), dejando 21 árboles en pie para su evaluación a los 10 años. De cada árbol se tomaron 2 trozas de 3,20 m: la basal (T1) y la segunda troza (T2). Se analizaron 38 trozas a las que se les estimó el módulo de elasticidad dinámico (MOE) por vibración longitudinal de las ondas de sonido, con una computadora portátil con el analizador de sonido con transformada rápida de Fourier FFT (Fakopp 
Enterprise, 2013). Este equipo captura la señal sonora y la descompone de forma individual en una gráfica de espectro (Sinais ingeniería, 2016). Para el cálculo del MOE se tomó el valor de densidad de madera de eucalipto en verde de 882 $\mathrm{kg} \mathrm{m}^{-3}$ (Sánchez-Acosta, 1987).

Se evaluaron 124 tablas de 3,10 m de largo (39, 43 y 42 tablas obtenidas del procesamiento de trozas de árboles de las clases de daño a, b y c, respectivamente). En la peor cara de cada tabla se registró presencia de médula y de defectos: grietas (Fig. 2a), bolsas y venas de kino (Fig. 2b, 2c y 2d), y pudrición (Fig. 2e y 2f).

Los promedios de $\mathrm{H}$ y DAP de los árboles medidos en 2012 y 2014 y los valores de MOE de las trozas se analizaron mediante técnica de modelos mixtos (MLGyM) y prueba LSD de Fisher de diferencias de medias. La asociación entre los daños por heladas y los defectos en tablas aserradas (grietas, kino, pudrición) se estudió mediante análisis de correspondencia múltiple y modelos lineales generalizados mixtos (MLGM) (Gbur et al., 2012), considerando la presencia de médula como defecto asociado al tamaño del árbol. Los MLGM permiten estudiar variables discretas y categóricas, donde los valores esperados de la variable respuesta se vinculan con las variables explicativas, expresadas como un modelo lineal, mediante una función de enlace. Los análisis estadísticos se hicieron utilizando los módulos MLGyM, MLGM y análisis multivariado del software InfoStat (Di Rienzo et al., 2015).

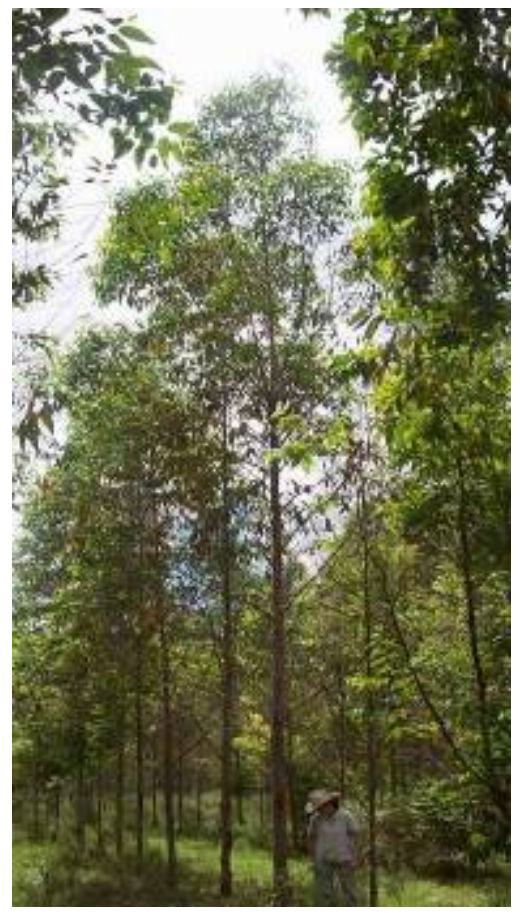

a)

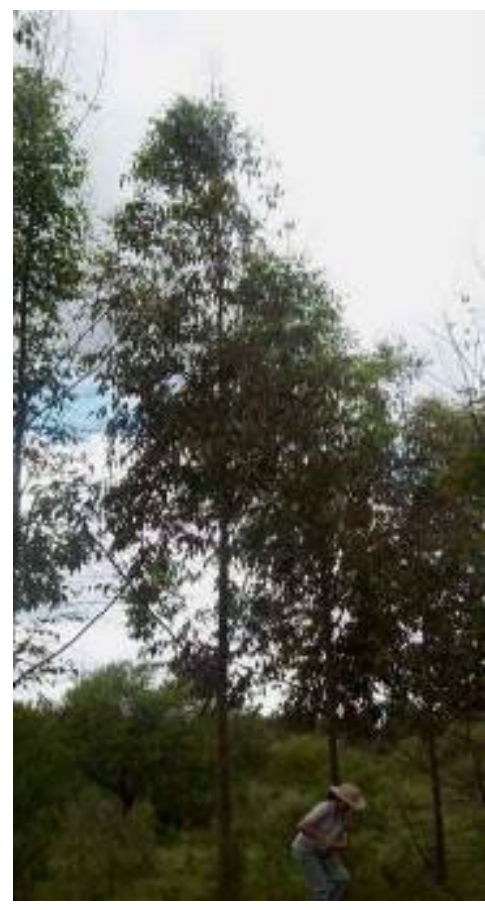

b)

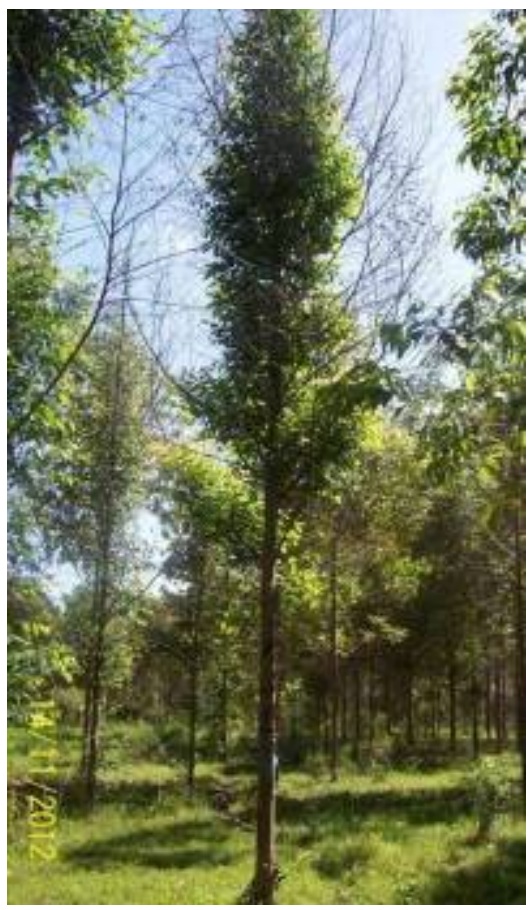

c)

FIGURA 1. Clases de daño por heladas en árboles según escala visual: a) copa sana, sin daño aparente por heladas, b) ápice seco, resto de la copa sin daño por heladas, c) copa totalmente seca, rebrotando algunas ramas o el fuste. 

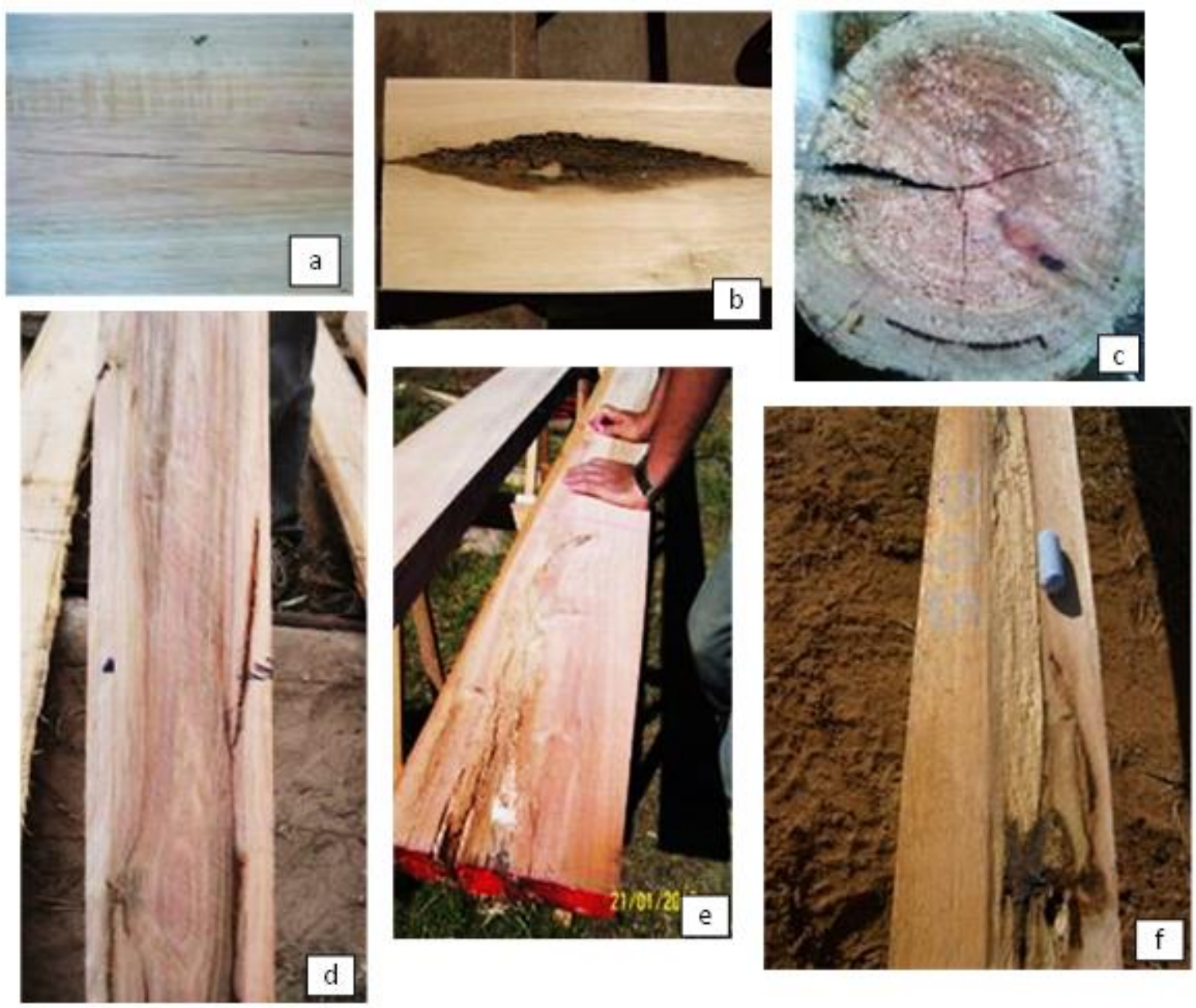

FiguRA 2. Daños encontrados en trozas y tablas aserradas, provenientes arboles de E. grandis afectados por frío: 2a, grietas; 2b, 2c y 2d, bolsas y venas de kino; 2e y 2f, pudrición.

\section{Resultados}

En 2012 el DAP y la H promedio de los árboles sin daño aparente por heladas (clase de daño a) fueron significativamente superiores a los de los árboles de las clases de daño b y c. En 2014 la altura promedio presentó el mismo comportamiento, mientras que el DAP promedio fue significativamente menor en los árboles con toda la copa helada (clase c) (Tabla 1).

Los valores de MOE de T1 resultaron significativamente menores a los MOE de T2 $(\alpha=0,05)$. En cuanto a T1, los MOE no presentaron diferencias significativas entre los árboles con diferente clase de daño por heladas. En cambio, en T2 el MOE fue significativamente mayor en los árboles de la clase a con respecto a los de la clase c (Tabla 2).

\section{Asociación entre las clases de daño por helada y los defectos en las tablas}

$\mathrm{Al}$ analizar los defectos en las tablas con y sin médula, según la clasificación visual de los árboles afectados por la helada, se aprecian las diferencias en la proporción de defectos registrados en las tablas de árboles de las tres clases de daño por heladas (Fig. 3). Se destaca que en ninguna de las tablas de árboles sin daño aparente por heladas se observó pudrición. 
TABLA 1. Prueba de medias LSD de Fisher $(\alpha=0,05)$ de altura $(\mathrm{H})$ y diámetro normal (DAP) de los árboles clasificados según daño por heladas, medidos en noviembre de 2012 y mayo de 2014.

\begin{tabular}{|c|c|c|c|c|c|}
\hline \multirow[t]{2}{*}{ Аño } & \multirow{2}{*}{$\begin{array}{c}\text { Clase de } \\
\text { daño } \\
\mathrm{a}\end{array}$} & \multicolumn{2}{|c|}{$\begin{array}{c}\text { DAP medio } \\
(\mathrm{cm})\end{array}$} & \multicolumn{2}{|c|}{$\begin{array}{c}\text { H media } \\
(m)\end{array}$} \\
\hline & & 13,01 & $A$ & 12,66 & $A$ \\
\hline \multirow[t]{3}{*}{2012} & $\mathrm{~b}$ & 11,49 & B & 10,94 & \\
\hline & C & 11,30 & B & 10,79 & \\
\hline & a & 17,59 & $A$ & 17,69 & $A$ \\
\hline \multirow[t]{2}{*}{2014} & b & 16,40 & $A$ & 15,75 & \\
\hline & C & 15,25 & B & 15,14 & \\
\hline
\end{tabular}

(a) copa sana, sin daño aparente por heladas, b) ápice seco, resto de la copa sin daño por heladas, c) copa totalmente seca, rebrotando algunas ramas o el fuste.

Medias con una letra común no son significativamente diferentes ( $p<=$ $0,05)$

TABLA 2. Promedio de los MOE por troza y clases de daño de los árboles según la escala visual definida.

\begin{tabular}{cccc}
\hline Troza & Clase de daño & MOE (GPa) & \\
\hline 1 & a & 8,61 & A \\
& b & 7,98 & A \\
& c & 7,52 & A \\
\hline 2 & a & 9,64 & A \\
& b & 8,92 & A B \\
& c & 8,17 & B \\
\hline
\end{tabular}

a) copa sana, sin daño aparente por heladas, b) ápice seco, helado, resto de la copa sin daño por heladas, c) copa totalmente seca, rebrotando algunas ramas o el fuste.

Medias con una letra común no son significativamente diferentes $(p<=$ $0,05)$

El análisis de correspondencia múltiple (Fig. 4) mostró asociación entre ausencia de médula, presencia de kino y árboles de la clase a; los árboles de la clase c mostraron asociación con presencia de grietas y pudrición. Los que no mostraron ninguna asociación fueron los árboles con daño únicamente en el ápice.

Solamente en árboles de las clases b y c se observó pudrición. Por lo cual, al estudiar la asociación de las clases de daño por helada y la presencia de pudrición solo se analizaron los datos referidos a tablas de árboles con daño del ápice y de toda la copa. Las asociaciones entre pudrición con las dos clases de daño por heladas con daños visibles $(\mathrm{p}=0,34)$ y con la presencia de médula $(\mathrm{p}=0,76)$ y su interacción $(p=0,069)$ no fueron significativas; además, la probabilidad de que aparezca pudrición no es significativamente diferente en tablas de árboles con daño del ápice con respecto a las obtenidas de árboles con toda la copa dañada por heladas.

En cuanto a las venas y bolsas de kino, la asociación con la presencia de médula en las tablas resultó significativa $(\mathrm{p}=0,0257)$, mientras que con la clase de daño por helada y la interacción de estas variables las asociaciones no fueron significativas ( $p=0,1083$ y $p=0,952$, respectivamente). Por otra parte, la probabilidad de encontrar kino cuando la tabla tiene médula es de $16 \%$, mientras que la probabilidad de encontrar kino si la tabla no presenta médula es de 34\%, y esas diferencias son estadísticamente significativas. Estos defectos aparecieron en árboles de las tres clases de daño definidas (Tabla 3).

Con respecto a las grietas, la asociación no resultó significativa con la clase de daño por heladas $(\mathrm{p}=0,38) \mathrm{ni}$ con la presencia de médula ( $\mathrm{p}=0,81$ ); las tablas provenientes de árboles de las tres clases de daño presentaron este defecto.

TABLA 3. Proporción de tablas con venas y bolsas de kino y superficie de las tablas afectada por ese defecto, separados de acuerdo con la clase de daño por heladas (a, b, o c) según se clasificaron los árboles de los que se obtuvieron las tablas.

\begin{tabular}{ccccc}
\cline { 2 - 5 } & \multicolumn{4}{c}{ Clase de daño } \\
\cline { 2 - 5 } & a & b & c \\
\hline Tablas con kino $\begin{array}{c}\text { (\% del } \\
\text { total) }\end{array}$ & 35,8 & 16,3 & 21,4 \\
$\begin{array}{c}\text { Superficie promedio afectada } \\
\text { (\% de la tabla) }\end{array}$ & 8,6 & 8,9 & 6,1 \\
\hline
\end{tabular}


Tablas sin médula

helada solo el ápice sin daño aparente helada toda la copa
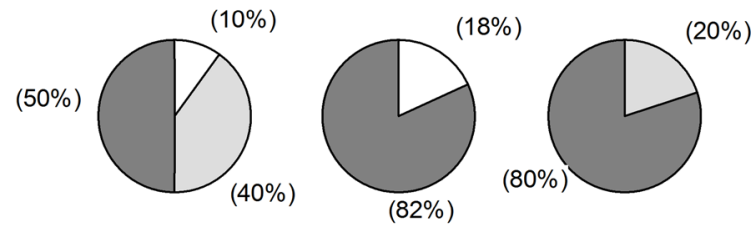

Tablas con médula

helada solo el ápice

$(50 \%)$

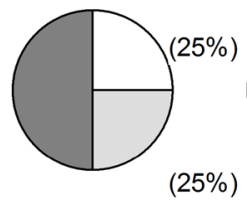

$\underline{\text { sin daño aparente }}$

helada toda la copa

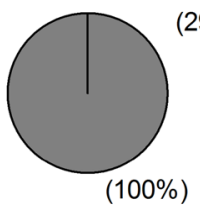

$(29 \%)$

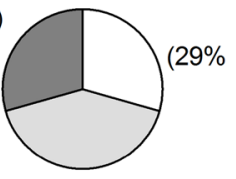

$(41 \%)$

grietas $\square$ pudrición $\square$ kino

FIGURA 3. Defectos para cada clase de daño por heladas, según escala visual aplicada a los árboles de los que provienen las tablas, para las tablas sin médula y con médula.

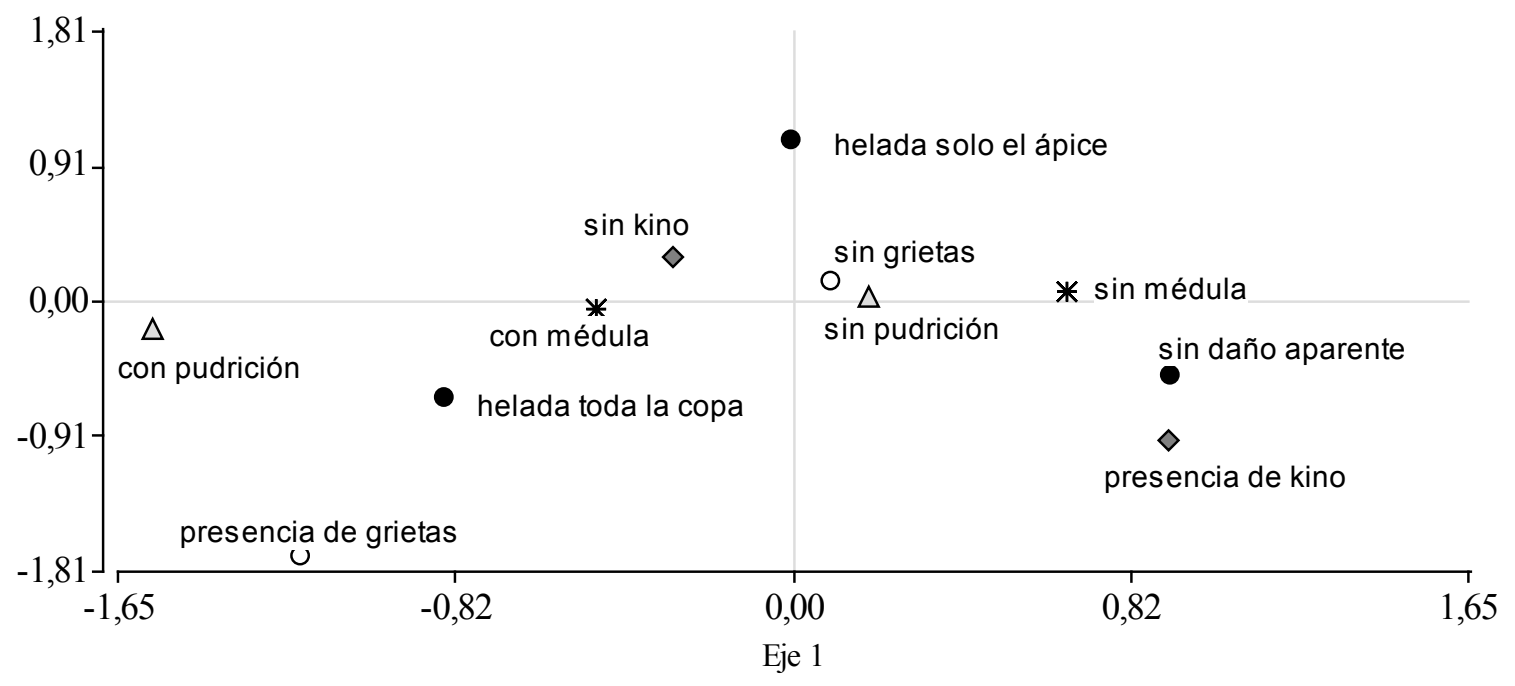

grado de daño por helada
kino
grietas $\triangle_{\text {pudrición }}$

FIGURA 4. Análisis de correspondencia múltiple entre la escala visual de daños por helada y los defectos en las tablas aserradas.

\section{DISCUSIÓN}

En este estudio los árboles de mayor tamaño no presentaron daños visibles por efecto de la helada; al contrario de lo que ocurrió con los árboles de menor tamaño, en los que se observaron daños en el ápice y en la copa por efecto de la helada al momento de la clasificación.
Además, los que sufrieron daño en toda la copa crecieron menos en diámetro en los años posteriores a la ocurrencia de las heladas. De acuerdo con los resultados de Gurskaya y Shiyatov (2006), los daños por heladas dependen del diámetro y del espesor de la corteza: los árboles de menor diámetro y con corteza más delgada sufren más el efecto de 
las heladas.En cuanto al menor valor de MOE de las trozas basales con respecto a las segundas trozas, otros estudios obtuvieron el mismo resultado. Xu y Walker (2004) y Soto, Valenzuela y Lasserre (2012) también encontraron en pino radiata de 27 años y 28 años valores de MOE de las segundas trozas siempre mayores que los de las primeras, en trozas de $5 \mathrm{~m}$ tomadas desde la base de los árboles. En las trozas basales los valores de MOE no resultaron diferentes para árboles con distinto daño por heladas probablemente debido al espesor de la corteza de las trozas basales y a la posición en el árbol; pero en las segundas trozas, las diferencias en el MOE de los árboles según la clase de daño sugiere que a mayor altura los daños por heladas son más notables. Los resultados de Stöckli y Schweingruber (1996a, 1996b) y Gurskaya y Shiyatov (2006) indican que los daños en la madera se evidencian desde el ápice hacia abajo. Los mismos autores encontraron, en estudios sobre abeto y alerce, que a partir de un cierto diámetro y espesor de corteza no aparecían más daños en el cambium a causa de heladas, explicando que la acumulación de calor durante el día es mayor cuanto mayor el diámetro, permitiendo mantener durante la noche la temperatura interior por encima de cero. Y como el diámetro del fuste y el espesor de la corteza van disminuyendo desde la base hacia el ápice, es a mayor altura del fuste donde deberían aparecer los mayores daños en la madera. Sin embargo, la altura de poda y el largo de corte de las trozas serán, para cada sistema productivo, las variables que permitan estimar la incidencia del daño por heladas en la producción de madera y el rendimiento industrial, de acuerdo con el destino de los rollos: la pérdida de rendimiento por saneamiento de tablas es mayor en los primeros rollos, destinados a aserrado y secado para obtener madera de alta calidad.

Las venas y bolsas de kino se observan en áreas próximas a lesiones en la madera (Shield y Mastrandrea, 2007), que entre otras causas pueden estar relacionadas al efecto de las heladas, como células aplastadas o deformadas (Stöckli y Schweingruber, 1996a, 1996b; Gurskaya y Shiyatov, 2006) por congelamiento del agua dentro y fuera de las células luego de un rápido descenso de la temperatura (Snyder y de Melo-Abreu, 2010).

Por otra parte, la presencia de kino puede indicar una respuesta de defensa a lesiones en la madera relacionadas con el efecto de diversos factores externos como las heladas (Wilkes, 1986; Jacobs 1986; Hillis, 1987). Las propiedades antisépticas de los taninos condensados presentes en el kino (Núñez, 2011) probablemente evitaron el ingreso de patógenos causantes de pudrición y no se registró ese defecto en ninguna tabla de los árboles sin daños visibles.

En las tablas de árboles de menor tamaño y con daños visibles por heladas, los defectos más frecuentes fueron grietas y pudrición, que puede hacerse evidente varios meses después de las heladas donde hay células dañadas por frío (Broschat, 2013). También las grietas en las tablas pueden formarse por daños anatómicos causados por las heladas (Pearce, 2001; Gurskaya y Shiyatov, 2006; Schweingruber, 2007) y aparecer aún en tablas de árboles sin daños visibles por frío, tal como se observó en este estudio.

Aunque Crane (2010) recomienda evaluar el daño por heladas de 2 meses a 6 meses luego de su ocurrencia, y en este estudio la clasificación de los árboles según la escala visual de daño por heladas se hizo cuatro meses después de su ocurrencia, mientras que la evaluación de defectos en la madera se realizó dos años después, el uso de esta escala visual para clasificar eucaliptos expuestos a heladas probablemente debería aplicarse inmediatamente después del evento meteorológico y realizar un seguimiento de la evolución de los síntomas visibles en los meses siguientes.

\section{CONCLUSIONES}

El daño por heladas según la escala usada se asocia con el módulo de elasticidad de las trozas y con presencia de pudrición en la madera. Sin embargo, no se asocia con otros defectos.

Si bien esta escala podría ser útil para tomar decisiones de manejo antes del turno, como cortar y conducir el rebrote, o ralear, se deben llevar a cabo otras investigaciones para determinar con mayor precisión los daños causados por frío en la madera aserrada y bajo 
heladas menos intensas, que son las que ocurren frecuentemente durante la época invernal en la región de estudio, así como aspectos metodológicos en relación al momento de la evaluación del daño en los árboles en pie.

\section{REFERENCIAS}

Alonso-Peña, J. R. (2011). Manual de histología vegetal. Madrid México: Ediciones Mundi-Prensa.

Broschat, T. K. (2013). Cold Damage on Palms. ENH-92, Series of the Environmental Horticulture Department, University of Florida, IFAS Extension. Recuperado de http:/ / edis.ifas.ufl.edu

Crane, J.H. (2010). Cold protection for tropical-subtropical fruit crops in Florida home landscape. University of Florida, IFAS Extension. Recuperado de http:/ / trec.ifas.ufl.edu/PDFs \% 20\&\%20Docs/Cold $\% 20$ protection $\% 20$ of $\% 20$ tropicalsubtropical $\% 20$ fruit $\% 20$ trees $\% 20$ in $\% 20$ the $\% 20$ home $\% 20$ landscape $\% 202011$.pdf

Del Tufo, N. (Octubre de 2012). Evaluación del raleo en un rodal afectado por heladas. En INTA-Aianer (Eds.), XXVI Jornadas Forestales de Entre Ríos. Concordia, Argentina: INTA-Aianer.

Di Rienzo, J., Casanoves, F., Balzarini, M., González, L., Tablada, M., \& Robledo, C. (2015). InfoStat versión 2015. Grupo InfoStat, FCA, Universidad Nacional de Córdoba, Argentina. Recuperado de http://www.infostat.com.ar

Fakopp Enterprise. (2013). Aplicación en planilla Excel de analizador de sonido con transformada rápida de Fourier (FFT). Recuperado de www.fakopp.com

Garrán, S., \& Garín, R. (2007). Las heladas de julio de 2007 en la región de Concordia. Sistema FruTIC. Recuperado de http:/ / comunidad.frutic.org.ar/foro/foro/textos/Las $\% 2$ Oheladas $\% 20$ de $\% 20 j u l i o \% 202007 \% 20$ en $\% 20$ Concordia.p df

Gbur, E., Stroup, W., McCarter, K., Durham, S., Young, L., Christman, M., West M., \& Kramer, M. (2012). Analysis of Generalized Linear Mixed Models in the Agricultural and Natural Resources Sciences. Madison Wisconsin, USA: American Society of Agronomy, Crop and Soil Science Society of America Inc. doi: 10.2134/2012.generalized-linear-mixedmodels

Gurskaya, M., \& Shiyatov, S. (2006). Distribution of frost injuries in the wood of conifers. Russian Journal of Ecology, 37(1), 712. doi: 10.1134/S1067413606010024
Hillis, W. (1987). Heartwood and tree exudates. Berlin, Germany: Springer-Verlag. doi: 10.1007/978-3-642-72534-0

Jacobs, M. (1986). Growth habits of the Eucalypts. Canberra, Australia: Forestry and Timber Bureau.

Núñez, C. (2011). Pulpa y Papel I (Décima parte), Química de la madera: Extractivos. Textos Técnicos: Madera y Pulpa. Recuperado de http://www.cenunez.com.ar/archivos/66PulpayPapelIDcimaParte.pdf

Pearce, R. S. (2001). Plant freezing and damage. Annals of Botany, 87 (4), 417-424. doi: 10.1006/anbo.2000.1352

Peña, C. de la, Flores, M. \& Ramos, S. (2012). Pérdidas económicas causadas por las heladas del 7, 8 y 9 de Junio en forestaciones de eucalipto de la costa del río Uruguay. Recuperado de https://inta.gob.ar/sites/default/files/script-tmp-inta_ev aluacin_econmica _.prdidas_heladas_junio_2012.pdf

Prado, J. A. (1989). Selección de especies y procedencias. En J. A. Prado \& S. Barros Asenjo. (Eds.), Eucalyptus: principios de silvicultura y manejo (pp: 42-56). Santiago, Chile: Instituto Forestal. Recuperado de http://biblioteca.infor.cl/dat afiles/8640.pdf

Sánchez Acosta, M. (1987). Relación peso-volumen en madera rolliza. Incidencia de la corteza. En INTA Concordia. (Ed.), Información Forestal, G. Explotación (pp. 1-3). Concordia, Argentina: Estación Experimental Agropecuaria INTA Concordia.

Schweingruber, F. H. (2007). Modification of the tree-ring structure due to extreme site conditions. En T. E. Timell \& R. Wimmer (Eds.). Wood structure and environment, Springer Series in Wood Science (pp. 87-92). Berlin - Heidelberg, Germany: Springer-Verlag. doi: 10.1007/978-3-540-485483

Shield, E. \& Mastrandrea, C. (2007). Reglas de tipificación de madera aserrada y secada en horno de Eucalyptus grandis en la Mesopotamia, Argentina. Concordia, Argentina: Proyecto Socodevi-INTA, Profeder y Pnfor 2211. Recuperado de www.biblioteca.org.ar/libros/210836.pdf

Sinais (2016). Curso de análisis de vibraciones. Recuperado de http://www.sinais.es/Recursos/Curso-vibraciones/funda mentos/transformada_fourier.html.

Snyder, R. L. \& de Melo-Abreu, J. (2010). Protección contra las heladas: fundamentos, práctica y economía (Vol. 1), Serie FAO. Recuperado de http://www.fao.org/docrep/012/y722 3s/y7223s.pdf

Soto, L., Valenzuela, L. \& Lasserre, J. (2012). Efecto de la densidad de plantación inicial en el módulo de elasticidad 
dinámico de árboles en pie y trozas de una plantación de pino radiata de 28 años, en la zona de arenales, Chile. Ciencia y Tecnología, 14(2), 209-224. doi: 10.4067/S0718221X2012000200008

Stöckli, V. \& Schweingruber, F. (1996a). A common mechanism for observed similarities in xylem abnormalities in response to frost drought transplantation and coppicing. En Stöckli, V. (Ed.), Tree rings as indicators of ecological processes: the influence of competition frost and water stress on tree growth size and survival (pp. 29-42). Disertación doctoral, Universität Basel, Basilea, Suiza.

Stöckli, V. \& Schweingruber, F. (1996b). Mechanism, anatomy and consequences of frost ring formation in mountain pine (Pinus mugo ssp unicata). En Stöckli, V. (Ed.), Tree rings as indicators of ecological processes: the influence of competition frost and water stress on tree growth size and survival (pp. 11-28). Disertación doctoral, Universität Basel, Basilea, Suiza.

Wilkes, J. (1986). Host attributes affecting patterns of decay in a regrowth eucalypt. V. Barrier zones. Holzforschung, 40(1), 37-42. doi: 10.1515/hfsg.1986.40.1.37
Xu, P. \& Walker, J. (2004). Stiffness gradients in Pinus radiata trees. Wood Science and Technology, 38(1), 1-9. doi: 10.1007/s00226-003-0188-2

Manuscrito recibido el 24 de noviembre de 2016

Aceptado el 12 de abril de 2018

Publicado el 25 de octubre de 2018

Este documento se debe citar como:

García, M. A., \& Mastrandrea, C. A. (2018). Defectos en madera de eucalipto asociados a daños por heladas en Entre Ríos, Argentina. Madera y Bosques, 24(3), e2431465. doi: 10.21829/myb.2018.2431465

\footnotetext{
Madera y Bosques por Instituto de Ecología, A.C. se distribuye bajo una Licencia Creative Commons Atribución-NoComercialCompartirlgual 4.0 Internacional.
} 